\title{
Blinded by the light
}

\author{
Anonymization should be used in peer review to prevent bias, not protect referees
}

\author{
David M Shaw
}

$\mathrm{P}$ eer review is an essential part of the research process, but there is considerable discussion about the quality and fairness of different peer review models. It is a widely accepted practice in the humanities to blind reviewers to the identity of authors, and this attitude is beginning to take hold in the biosciences and medicine too; Nature Publishing Group recently announced that it would join many other journals in the humanities and natural sciences that offer double-blind review, in which neither the authors nor the reviewers know each other's identities [1]. Making authors anonymous is certainly important [2], but agreement on this point has led to the widespread assumption that reviewers should also be anonymous. In this article, I analyse the pros and cons of different blinding systems, before arguing that only author identities should be anonymized and that blinding authors to the identities of reviewers is unethical.

The double-blind peer review system is regarded by some as the fairest model for all parties involved and is used by many journals. It has one major advantage and one minor one. First, by blinding reviewers to the identity of authors, it ensures that reviewers cannot be biased on account of the author's sex, home country, lack of seniority or publication record. It also prevents "big names" from trading on their reputation-unless the reviewer can work out who the author is because of telltale signs in the manuscript. To avoid this, some journals require authors to remove all of their own papers from the references, but a reviewer can still sometimes find out the authors identities [3]. Nonetheless, deliberately revealing the authors' identities is virtually an invitation to be biased.

Vice versa, blinding authors to the identities of reviewers ensures that reviewers can give an honest assessment of the manuscript without fear of retaliation, in particular if the author is a senior and influential figure in the field. However, this supposed advantage has negative effects that mitigate its perceived benefits. The flipside of protecting reviewers is lack of accountability. Although reviewers are accountable to editors, they are not accountable to authors, and anonymizing reviewer identities means that authors are often left without recourse when a review is unfair-they cannot point out that the reviewer had a conflict of interest that went undetected by an editor, for example. Some journals allow authors to exclude specific scientists from reviewing their manuscripts, but they usually limit this option to only one or two names, even though more potential reviewers might have a possible conflict of interest. While blinding reviewers to author identity is essential to prevent both deliberate and accidental bias, blinding authors to reviewer identity generates a power imbalance between the two: given that reviewers recommend whether papers should be published or not, transparency dictates that their identities should be known, even if the editor makes the final decision.

\section{"...only author identities should be anonymized [...] blinding authors to the identities of reviewers is unethical."}

Most journals that do not use the double-blind system operate a different model, which reveals the authors' identities to reviewers, whereas reviewers remain anonymous. This system dispenses with the major advantage of double blinding and maintains its main disadvantage: reviewers remain unaccountable to authors and the wider community, which enables unethical reviewers to act on any personal biases-perhaps against women or lesserknown researchers-while hiding behind anonymity. This might be more of a problem for journals in the humanities, where intellectual conflicts of interest are perhaps more common, but it could also affect biomedical journals. Most major molecular biology journals use this anonymous reviewer model, although some, including the EMBO Press journals and the medical journal The Lancet, do allow reviewers to sign their reviews if they wish to do so. It appears unlikely that many reviewers will take advantage of this opportunity, for reasons discussed below.

"...authors should benefit from the protection of anonymity, as they are the ones who are affected by potential bias..."

A third option is the non-blinded, open review model: reviewers know who the authors are and authors know who the reviewers are. While this has the advantage of making reviewers accountable, it nonetheless retains the problem that reviewers can still be biased against certain authors [2].

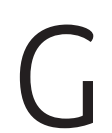
iven the preceding analysis, it appears that the best option is single-blind review in favour of the authors: authors know who the reviewers are but the reviewers do not know who the authors are-at least until a paper has been published [4]. One potential disadvantage of 


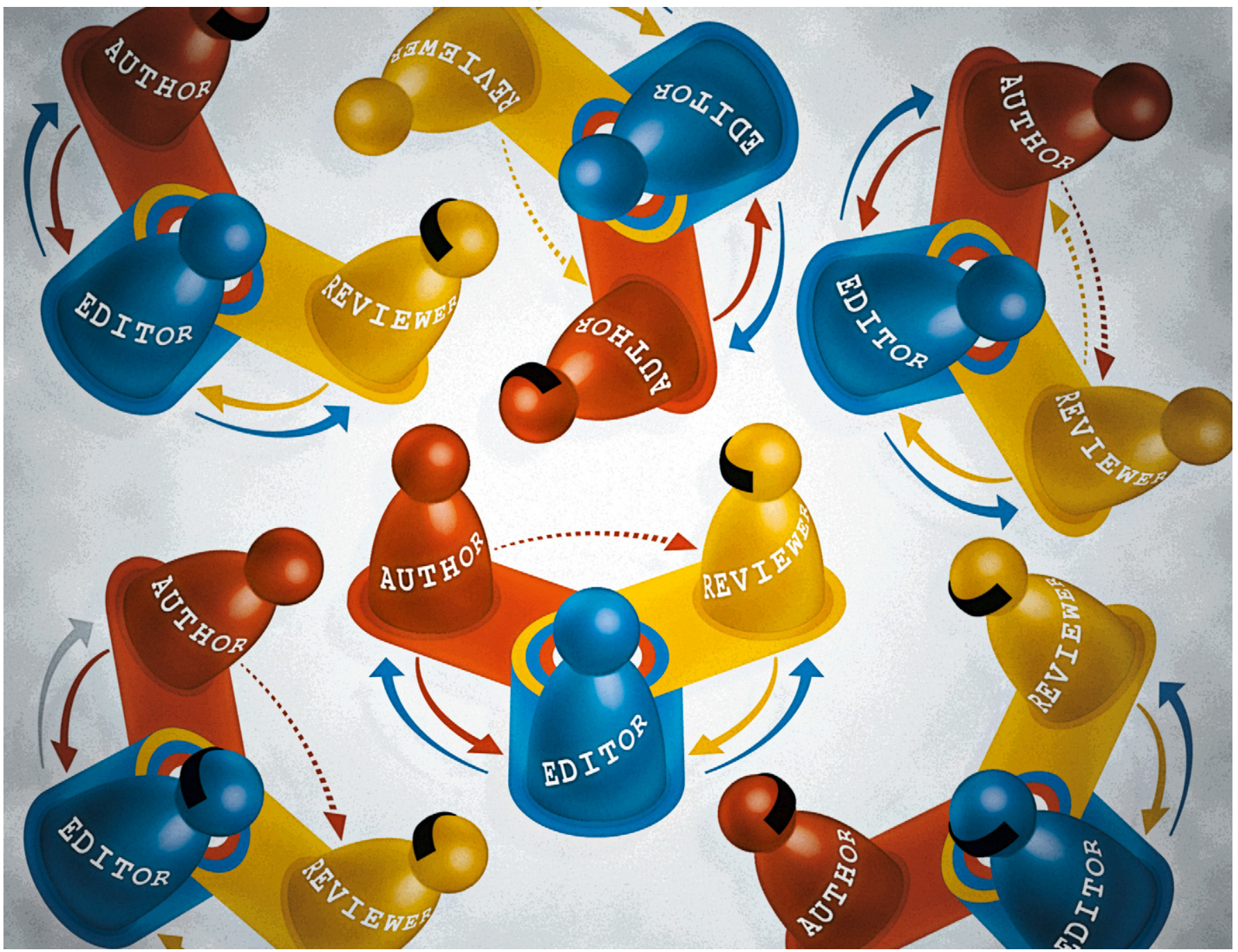

single-blind review is that authors could bear a grudge about a bad review and might potentially discriminate against their reviewers at some point in the future. However, they cannot do anything retaliatory in terms of reviewing their reviewers' future work negatively if all journals adopt this policy, as they will not know whether the nasty reviewer is the author they are reviewing; nonetheless, retaliation could take place via other avenues such as hiring or funding decisions. However, anyone who has negatively reviewed some senior person's manuscript-they will know they have, because the author identities are revealed at acceptance or publication-could always point out the conflict of interest if that person is involved in hiring procedures and does not declare the conflict.

One putative disadvantage of this proposal is that, in some fields, it might deplete the pool of available and knowledgeable reviewers: sooner or later, most of the top people in a given field will have reviewed each other's papers and will have to recuse themselves from reviewing papers by someone they have themselves been negatively reviewed by. But this objection is based on two misconceptions. First, excluding experts from review panels relates to grant applications and hiring decisions, not to reviewing research manuscripts-under this system, no one knows the identity of the authors, and so no one can be biased. Second, the suggestion is not that experts recuse themselves, but that they declare a potential conflict of interest such as "this person gave me a really terrible review once" in these specific contexts. And third, blinding of authors or applicants, except at the interview phase, should be universal: it is hardly a fair objection to a proposal to anonymize authors that such a proposal would run into future problems at any journal, university or funding agency that did not adopt a policy of anonymizing authors or applicants.

The potential for retaliation against identified reviewers may mean that some junior researchers are less likely to review manuscripts, but they already bear the burden of reviewing anyway and for them getting published is more important than reviewing. Another reason in favour of blinding reviewers to author identities and requiring reviewers to sign their reviews is that reviewers will eventually find out who wrote a paper, whereas under double-blind peer review or the current anonymous reviewer system, the authors will most likely never find out who reviewed their paper. In this sense, double blind is not 
symmetrical at all; it is slanted in favour of reviewers.

Reviewers could also benefit from being identified: if their names are printed alongside the published article, they receive some credit for the substantial contribution they made to improve a paper. Furthermore, the fact that their identities are revealed is likely to act as an incentive to ensure that reviews are thorough and fair, thereby increasing transparency and the overall quality of the peer review process. An alternative way of providing a similar incentive would be to publish both the reviewer names and the reviews themselves. Some journals, including the EMBO Press journals, already publish peer reviews in full alongside the published article, but only a few, such as BMJ Open, include the names of the reviewers. Although this approach has several advantages, it might have even more of a negative effect on reviewer recruitment than merely revealing their names would have. Two other points are worth mentioning here: first, if a reviewer improves a paper immensely, it could potentially be embarrassing for the authors to have the review published alongside their finished article; second, it is even possible that such a reviewer could meet formal criteria for authorship [5].

\section{". . mild bias in favour of suspected prominent researchers is much less important than strong bias against authors who are not "big names"."}

Assuming that the single-blind system is adopted, should authors be able to opt out of blinding if they wish to? No. Blinding must be mandatory because deanonymizing will allow authors with "big names" to trade on their reputation. While it can be obvious whether a paper was written by a "big name” because of the style or content, even if references are anonymized, this cannot be helped. But mild bias in favour of suspected prominent researchers is much less important than strong bias against authors who are not "big names". Furthermore, a system where reviewers must try to work out the identities of authors through guesswork or literature searches is clearly less prone to bias than one where the author identity is revealed up front.

\section{". . another issue that has been widely neglected in the peer review debate is that bias can also affect editors."}

Reviewers should not be able to opt out of signing either, as they must remain accountable. This could make it more difficult for journals to attract reviewers, but it would be worth it to improve the peer review system. Allowing reviewers to suggest alternative colleagues to review a paper as some journals do gives them too much influence over a supposedly impartial process. Reviewers should sign reviews so that authors know who is rendering an assessment.

t should be noted that the double-blind system does not offer total anonymity to authors; another issue that has been widely neglected in the peer review debate is that bias can also affect editors. Some journals have a triple-blind review system where editors are also blind to the identity of authors - but obviously not to the identity of reviewers, who they must invite. Is this better than double-blind, or than reviewerblind models? Editors too should remain unaware of the identity of authors, as many submissions are rejected without review, and even well-intentioned editors are subject to unconscious bias, just as reviewers are. If an editor receives a paper with the name of one of the most famous researchers in the world on the front, he or she might be more likely to give the paper the benefit of the doubt and send it out for review, even if it is of borderline interest, than if it were written by a novice. Furthermore, incidents have been reported of editors in the humanities using authors' identities to check their CV, education and previous publications before deciding whether to reject without review: "in the internet age, googling to find out [about] the authors of papers just seems too tempting for editors and reviewers" (http://dailynous.com/2015/02/02/guardingthe-guardians-or-editors/). I myself have been discriminated against, despite being an established researcher: a major bioethics journal imposed a limit of one paper per year on me because they had published too many of my papers; I grudgingly complied. I have not heard of life science journals behaving in this way, but it would not surprise me to hear similar stories from colleagues in other disciplines.

\section{"The flipside of protecting reviewers is lack of accountability.}

But triple blind is the wrong solution for the same reasons that double blind is: both involve reviewer anonymity. Actually, the best option is not single-blind, but a different type of double-blind review: the editor and reviewer are blind to the identity of the author, but the author knows who the editor and the reviewers are. It might be objected that this puts too much power in the hands of authors, but this transparency actually offers little potential for abuse. It is normal for authors to know who the editors are, and informing them of reviewers' identities when reviews come in does not give authors any opportunity to influence the decision about their manuscript. In fact, the main drawback of blinding editors to authors' identities is that they would be unable to avoid inviting reviewers who work in the same department as the authors or who have some other personal conflict of interest, but safeguards could be implemented to avoid these problems. First, it is normally up to reviewers to report conflicts of interest anyway, and publication of reviewer names alongside final articles would make it obvious if anything unethical had happened. More importantly, any editorial bias is likely to be most significant at the initial stage of deciding whether to send a paper for review and editors could be unblinded when reviews come in in order to check for any potential problems or conflicts. Furthermore, the system could be set to exclude reviewers from the same institution automatically if an editor selected one. Another potential pitfall of having editors blinded to authors is that they could theoretically select an author to review her own work. But safeguards against such problems can also be developed: for example, electronic submission systems could issue alerts if a selected reviewer had the same email address as an author. While this would have the 
disadvantage of unblinding, this would only happen once the paper had already been selected for review.

In conclusion, it is so widely recognized that authors' identities should be hidden from reviewers that this glaring truth has blinded many to the fact that it is almost as important to disclose reviewers' identities. Reviewers and editors make decisions about authors, but authors do not make decisions about reviewers or editors; this means that authors should benefit from the protection of anonymity, as they are the ones who are affected by potential bias - and this protection is lifted upon publication in any case.
Reviewers and editors have far more power than authors, so an asymmetry in blinding between them that favours authors is clearly justified. In fact, the peer review system should be based upon the principle that blinding should be used only to prevent bias in decision-making.

\section{Conflict of interest}

The author declares that he has no conflict of interest.

\section{References}

1. Anon (2015) Nature journals offer double-blind review. Nature 518: 274
2. Schmid JA (2003) Cover the eyes of Lady Justice: an appeal for double-blind peer reviewing. EMBO Rep 4 : $734-736$

3. Chawla DS (2015) Nature to let potential authors try a double-blind date. Science doi:10.1126/science.aaa7888

4. Mulligan A, Hall L, Ellen R (2013) Peer review in a changing world: an international study measuring the attitudes of researchers. J Am Soc Inf Sci Tech 64: 132-161

5. Erren T, Erren M, Shaw D (2013) Peer reviewers can meet journals' criteria for authorship. BMJ 346: f166 